\title{
Les aspects biologiques de la fécondation in vitro
}

Environ mille cinq cents enfants sont déjà nés dans le monde, dont plus de cent en France, grâce à la fécondation in vitro et transfert d'embryons. La méthode permet actuellement d'obtenir des taux de succès seulement deux fois inférieurs à ceux de l'insémination naturelle.

\section{Jacques Testart Bruno Lassalle Monique Volante Joëlle Belaisch-Allart René Frydman}

\section{REFERENCES}

1. McBain JC, Gronow MJ, Bayly CM, Martin MJ. Controlled ovarian stimulation with clomiphene and HMG for in vitro fertilization in: Feichtinger W, Kemeter P. eds. Recent Progress in Human in vitro fertilization. Cof ese 1984 .

2. Frydman R, Testart J, Belaisch JC. Comparaison des différentes méthodes de monitorage de l'ovulation en vue d'une fécondation in vitro. Gynécologie 1982; 33-5:387-92.

\section{ADRESSE}

Unité Inserm U.187 et service de gynécologieobstétrique (Pr. E. Papiernik). Hôpital AntoineBéclère. 157 , rue de la porte-Trivaux. 9214I Clamart.

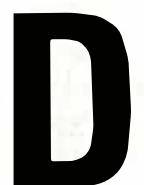

epuis la naissance de Louise Brown en 1978, plus de I 000 enfants ont vu le jour après Fécondation In Vitro Et Transfert d'Embryons (FIVETE) et on peut estimer à une centaine le nombre d'enfants qui naissent chaque mois dans le monde grâce à cette méthode. Cette accélération des résultats est la conséquence d'une plus grande efficacité technique mais aussi d'un accroissement considérable du nombre des équipes impliquées dans la FIVETE et du nombre des couples demandeurs.

Schématiquement la FIVETE consiste à recueillir un ou plusieurs ovules chez la femme, à les incuber au laboratoire, avec les spermatozoïdes du conjoint masculin, puis à introduire dans l'utérus de la même femme le ou les œufs fécondés, 2 jours plus tard. Pour augmenter les chances de succès lors de chaque cycle de tentative, on provoque artificiellement la maturation simultanée de plusieurs follicules ovariens par divers traitements hormonaux de la patiente, traitements qui commencent le plus souvent 2 à 5 jours après les dernières règles. Quand les follicules sont mûrs, l'ovocyte contenu dans chacun de ces follicules est lui-même amené à maturation (ovule) grâce à l'administration finale de l'hormone gonadotrophine chorionique (hCG). Cette hormone présente les mêmes propriétés que l'hormone lutéotrophe ( $\mathrm{LH})$ qui, dans un cycle naturel, est massive- ment déchargée par l'hypophyse vers les $12-13^{\mathrm{e}}$ jours et induit la rupture folliculaire 37 heures plus tard. Puisqu'il s'agit de recueillir l'ovule à l'intérieur même de son follicule, l'intervention prend place 35-36 heures après l'administration de hCG. Il est donc facile de programmer l'heure de la ponction folliculaire en décidant de l'heure d'injection de hCG; en pratique hCG est administrée le soir entre $2 \mathrm{I} \mathrm{h}$ et minuit et le recueil des ovules a lieu le surlendemain, entre $9 \mathrm{~h}$ et midi. Cet horaire arbitraire autorise les actes chirurgicaux et biologiques pendant les heures ouvrables. Ainsi l'heure d'intervention est laissée à la merci de l'équipe FIVETE mais le jour de cette intervention est fonction de la réponse au traitement des ovaires de la patiente (maturité des follicules). Le sperme du conjoint est recueilli par masturbation peu avant ou peu après les ovules de la patiente, selon les équipes. Sa préparation demande moins de I heure et on procède immédiatement à l'insémination in vitro. Certaines équipes effectuent le transfert du ou des oufs fécondés dès le jour suivant l'insémination mais la plupart préfèrent attendre la segmentation qui, dans nos conditions d'observation, est le premier signe incontestable de vitalité. La première division de l'œuf, qui conduit à deux blastomères, survient environ 30 heures après l'insémination; vers $40-46$ heures, l'œuf compte le plus souvent quatre blastomères, mais des nais- 
sances ont suivi le transfert d'œufs à deux ou huit blastomères à ce même moment. C'est une singularité de l'utérus humain d'accepter l'œuf seulement I jour ou 2 après l'ovulation, la gestation ne pouvant être établie chez les femelles animales que si l'œuf est transplanté plus de 2 jours après l'ovulation; cette singularité est un grand avantage dans la FIVETE car on est encore peu compétent pour cultiver l'œuf pendant plusieurs jours. On remarquera aussi que la fécondation in vitro a des résultats exceptionnels chez l'homme $(80 \%$ des ovules fécondés) comparables à ceux obtenus chez les rongeurs alors que la méthode est encore inefficace chez les grands mammifères domestiques (ovins, bovins, porcins).

Enfin, il est important de signaler que la "qualité" des enfants nés après FIVETE n'est pas altérée par rapport à la procréation naturelle (ni la proportion des enf ants de chaque sexe), il se pourrait même que la fréquence des anomalies à la naissance soit diminuée après FIVETE. Cette tendance, qui demande a être vérifiée avec des effectifs plus nombreux, peut trouver une explication : dans la FIVETE, ovules et spermatozoïdes sont mis en présence, avant tout vieillissement, dès que leur aptitude à produire un œuf normal est atteinte.

\section{L'obtention} des ovules

Les inducteurs de l'ovulation sont utilisés dans le cadre de la fécondation in vitro dans un double but : celui de programmer l'heure de la ponction folliculaire et celui de recueillir plusieurs ovocytes matures au cours d'une seule intervention chirurgicale.

\section{Stimulation}

Plusieurs schémas sont habituellement proposés pour stimuler la croissance folliculaire. On peut faire appel soit au citrate de clomiphène seul, soit au citrate de clomiphène associé à l'HMG (human menopausal gonadotropin), soit à l'HMG seul $[\mathrm{I}, 2]$. A cette stimulation ovarienne, il faut ajouter l'induction de l'ovulation par une administration d'hCG. Quelles que soient les habitudes de chaque équipe, des points fondamentaux sont désormais acquis. La stimulation ovarienne doit aboutir à l'obtention de plusieurs ovocytes car le transfert de plusieurs embryons augmente les chances d'établir une grossesse. L'adjonction d'HMG au clomiphène aboutit à un recrutement folliculaire supérieur et augmente la fécondabilité des ovocytes (tableau I). L'hCG est administré lorsque le taux d'œstradiol plasmatique est voisin de $400 \mathrm{pg} / \mathrm{ml}$ par follicule dont le diamètre $\mathrm{a}$ atteint $\mathrm{I} 6$ à $18 \mathrm{~mm}$ selon l'observation échographique. Le recueil d'ovocyte s'effectue $35 \pm$ I heure après l'injection d'hCG quelle que soit la dose administrée (5 000 ou 10000 U.I.).

Les niveaux hormonaux en phase lutéale ne sont pas altérés par les stimulations employées. L'analyse histologique de biopsies après HMG semble montrer un effet favorable de cette médication sur l'endomètre. La réponse ovarienne à la stimula- tion est variable selon les femmes, et au cours de différents cycles chez la même femme. L'adaptation de la stimulation à chaque cycle varie en fonction de la longueur du cycle, de la présence d'un ou de deux ovaires, de l'existence d'une dysovulation, d'une anovulation connue, de l'intolérance au clomiphène, ou d'une résistance à l'HMG lors des cycles précédents. Enfin d'autres modes de stimulation sont aujourd'hui envisagés : la FSH (hormone folliculostimulante) pure ou stimulation par LH-RH. Le monitorage de l'ovulation fait appel à la combinaison de deux techniques :

l'échographie qui permet d'enregistrer quotidiennement le nombre et la taille des follicules recrutés. Les follicules ovariens apparaissent comme des zones plus ou moins arrondies, anéchogènes, à contours nets. Les diamètres sont mesurés pour chaque follicule, la moyenne calculée reflète la taille réelle des follicules en croissance;

les dosages hormonaux : vers le ${ }_{10}{ }^{\mathrm{e}}$ jour du cycle traité, un prélèvement plasmatique quotidien pour dosage rapide d'œstradiol et de $\mathrm{LH}$ est effectué. La confrontation des résultats écho-biochimiques se fait le soir même, les patientes sont alors immédiatement averties de la poursuite du traitement inducteur, ou de l'injection d'hCG en fonction de la réponse ovarienne selon les critères précédemment cités. Les dosages de LH quotidiens permettent de s'assurer qu'il n'y a pas eu de décharge spontanée de cette gonadotropine hypophysaire qui entrainerait une incertitude dans le moment de la rupture folliculaire.

Tableau I

INFLUENCE DU TRAITEMENT DE STIMULATION SUR LA REPONSE OVARIENNE ET LE RESULTAT DE LA FIV

\begin{tabular}{|l|c|c|c|c|c|}
\hline traitement & $\begin{array}{c}\text { nombre } \\
\text { de cycles } \\
\text { avec } \\
\text { ponction }\end{array}$ & $\begin{array}{c}\text { nombre } \\
\text { moyen de } \\
\text { follicules } \\
\text { ponctionnés }\end{array}$ & $\begin{array}{c}\text { volume } \\
\text { folliculaire } \\
\text { (ml) }\end{array}$ & $\begin{array}{c}\frac{\text { embryons }}{\text { ovocytes }} \\
\text { (\%) }\end{array}$ & $\begin{array}{c}\text { embryons } \\
\text { par cycle }\end{array}$ \\
\hline clomiphène & 90 & $2,7 \pm 0,1$ & $5,2 \pm 0,2$ & 42 & $0,6 \pm 0,1$ \\
clomiphène+HMG & 217 & $3,8 \pm 0,1(a)$ & $4,8 \pm 0,2$ & $71(a)$ & $1,4 \pm 0,1(a)$ \\
\hline
\end{tabular}




\section{REFERENCES}

3. Frenkel Y, Oclsner G, Benbaruch G, Menczer J. Major surgical complications of laparoscopy. Europ 7 Obstet Gynec Reprod Biol 1981; 12 : 107-11.

4. Belaisch-Allart J, Guillet-Rosso F, Baton C, Champagne C, Bodereau AM, Frydman R. Caclioscopie sous anesthésie locale pour recueil d'ovocyte en vue d'une fécondation in vitro. Nouv Presse Méd 1983; 12 : 2053-4.

5. Lenz S, Lauristen JG. Ultrasonically guided percutaneous aspiration of human follicles under local anesthesia. A new method of collecting oocytes for in vitro fertilization. Fertil Steril 1982; 38 : 673-7.

6. Dellenbach P, Nisarid I, Moreau L, et al. Transvaginal, sonographically controlled ovarian follicle puncture for eggs retrivial. Lancet 1984; I : 1467 .

7. Belaisch-Allart JC, Hazout A, Guillet-Rosso F, Glissant M, Testart J, Frydman R. Various technics for oocyte recovery. I In Vitro Fert Embryo Transfer 1985; sous presse.

8. Testart J, Lassalle B, Frydamn R. Apparatus for the in vitro fertilization and culture of human oocytes. Fertil Steril 1982; $38: 372-5$.

9. Menezo Y, Testart J, Perrone D. Serum is not necessary for human in vitron fertilization, early embryo culture and transfert. Fertil Steril 1984; $42: 750-5$.

10. Veeck L, Worthman J, Withmyer J, et al. Maturation and fertilization of morphologically immature human oocytes in a program of in vitro fertilization. Fertil Steril 1983; $39: 594-602$.

II. Testart J, Lefevre B, Castanier M, BelaischAllart J, Guillet-Rosso F, Frydman R. Comparative effect of clomiphen and clomiphen + HMG on the preovulatory follicles and fertilizability of the oocyte. Ann NY Acad Sci 1985; sous presse.

12. Mahadevan M, Trounson A. The influence of seminal characteristics on the success rate of human in vitro fertilization. Fertil Steril 1984; $42: 400-5$.

13. Testart J, Belaisch-Allart J, Hazout A, Guillet-Rosso F, Frydman R. Actual methods
Pour le prélèvement ovocytaire, la cœlioscopie permet de visualiser l'ovaire ou les deux ovaires et l'utilisation d'une pince à ponction, par une troisième vioie, permet de présenter successivement l'aiguille de ponction aux différents follicules pré-ovulatoires.

De l'accessibilité et de la mobilité de chaque ovaire dépendent les possibilités de recueil d'ovocytes. Le pneumopéritoine est créé grâce à une insufflation de $\mathrm{CO}_{2}$ pur.

Plusieurs types d'aiguilles à ponction ont été proposés, le diamètre intérieur de l'aiguille varie de 1,2 à I, $6 \mathrm{~mm}$, sa paroi est parfois recouverte de Téflon; de même certains utilisent une aiguille à double courant afin de permettre le lavage immédiat du follicule. Le système d'aspiration varie de la simple seringue préchauffée à $37 U C$ adaptée à l'aiguille, au modèle plus sophistiqué où l'aspiration se fait sur commande d'une pompe à vide et où la pression est parfaitement mesurée. L'anesthésie générale au cours de la colioscopie n'est pas exempte de risques [3]. Afin de diminuer les accidents allergiques liés aux tentatives répétées, la cœlioscopie sous anesthésie locale a été préconisée, associée à une neuroleptanalgésie [4]. Tout en accordant les indications de cette méthode au désir des patientes, et à l'état de leur pelvis, il est certain que le développement du recueil d'ovocytes sous anesthésie locale va dans le sens d'une participation plus grande des patientes et d'un allègement du coût de la méthode par diminution de la durée d'hospitalisation.

Dans ce même ordre d'idées, le développement du recueil ovocytaire sous contrôle échographique [5] présente un certain nombre d'avantages : sa simplicité, et son moindre coût puisqu'elle évite l'anesthésie générale et l'hospitalisation, sa plus grande innocuité puisqu'elle supprime les risques inhérents à l'anesthésie et à la cœlioscopie, en fait sa répétabilité puisque les deux premiers avantages autorisent des tentatives plus fréquentes. Cette technique nécessite une prémédication, un sondage vésical et l'emploi d'aiguilles de 20 à $23 \mathrm{~cm}$ de longueur. Il existe des contre-indications : l'anxiété de la patiente, les adhérences digestives périovariennes, une infection urinaire récente. En cas d'ovaires inacessibles par voie transvésicale ou d'ovaires rétro-utérins, il est intéressant d'utiliser la voie transvaginale [6]. Ces différentes techniques permettent d'offrir un choix à la patiente en fonction de son désir et des particularités individuelles. Les taux de grossesse obtenus avec ces méthodes sont comparables [7].

\section{La phase in vitro}

C'est la période du cycle FIVETE pendant laquelle les gamètes sont préparés puis mis en contact in vitro jusqu'à l'obtention d'un embryon divisé deux jours plus tard.

Comme pour les espèces animales, la fécondation externe de l'ovule humain et la culture de l'embryon doivent s'effectuer dans des conditions d'environnement aussi proches que possible de celles qui existent dans l'appareil génital : température maintenue à $37^{\circ} \mathrm{C}$, obscurité, légère alcalinité $(\mathrm{pH}=7,3$ à 7,5$)$, pression osmotique maintenue vers $280-290$ milliosmoles $/ \mathrm{kg}$. Il existe actuellement deux types d'enceinte de culture. La plupart des groupes ont repris le matériel utilisé pour la FIV chez l'animal et qui réserve l'étude à $37^{\circ} \mathrm{C}$ aux périodes de culture, les manipulations s'effectuant à l'extérieur; nous avons proposé [8] de réaliser l'ensemble de la phase in vitro (culture et manipulations) à l'intérieur d'un incubateur spécialement équipé. Le modèle actualisé et disponible de cet incubateur (WildLeitz, France) permet de manipuler à $36 \pm 1^{\circ} \mathrm{C}$ et de cultiver gamètes et embryons à $37 \pm 0,1^{\circ} \mathrm{C}$.

La fécondation et la culture de l'œuf sont réalisées en boîtes ou plus souvent en tubes, dans des milieux synthétiques de composition variée. La plupart des groupes français utilisent le milieu $B_{2}$ de Menezo (APISystem) qui ne nécessite aucune préparation et surtout aucune addition de sérum sanguin [9]. L'utilisation d'un milieu entièrement synthétique, donc de composition parfaitement définie, n'est pas seulement d'intérêt pratique (simplification des préparations); il 
permet aussi une grande stabilité technique (le milieu est constamment identique) et autorise des études sur le métabolisme précoce de l'œuf (modifications dans la composition du milieu synthétique dans lequel l'œuf a été cultivé).

Les étapes successives de la FIV au laboratoire sont: la recherche de l'ovocyte dans le liquide aspiré de chaque follicule, la mise en culture immédiate dans les conditions précitées, puis la préparation des spermatozoïdes. Le sperme est dilué avec une solution saline et centrifugé deux fois. Le culot cellulaire est alors immergé avec du milieu de culture; les spermatozoïdes les plus mobiles s'échappent du culot et sont recueillis au sein du liquide surnageant. Ces manipulations ont pour but d'éliminer le plasma séminal qui inhibe la capacitation (acquisition de la fécondance) des spermatozoïdes, et de concentrer une partie des meilleurs gamètes dans la fraction utilisée pour l'insémination. Et enfin, l'insémination in vitro a lieu, I à 8 heures après le recueil ovocytaire. Le jour suivant on procède à la dénudation mécanique de l'œuf et à son transfert dans un milieu de culture identique mais dépourvu de spermatozoïdes. La dénudation, qui consiste à ôter les cellules folliculaires présentes autour de l'œuf permettra l'observation du développement ultérieur.

Le second jour les œufs divisés (le plus souvent au stade de quatre blastomères) sont transplantés dans l'utérus de la patiente.

\section{-Taux de succès}

Le taux de succès de la phase in vitro, exprimé comme la proportion d'œufs divisés parmi les ovocytes inséminés, dépasse $80 \%$ quand les gamètes disponibles sont de qualité satisfaisante.

La fécondabilité des ovocytes dépend de plusieurs facteurs : la nature du traitement de stimulation ovarienne : seulement la moitié des ovocytes recueillis sont immédiatement fécondables après stimulation avec HMG, mais une partie des ovocytes considérés comme "immatures sains", deviennent fécondables après douze à trente six

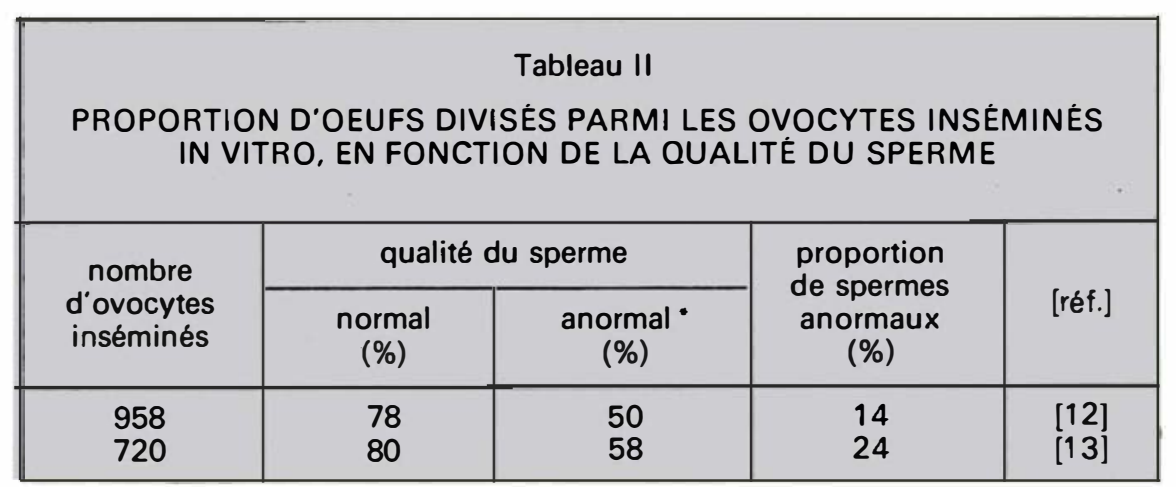

* <15-20 millions spermatozoïdes/ml; <50\% motilité; < 40-50 \% formes normales.

heures d'incubation in vitro [1o] La fécondabilité est améliorée si l'on adjoint HMG aux traitements par le clomiphène (tableau I);

(b) les caractéristiques des follicules ponctionnés : plusieurs paramètres folliculaires ont été analysés pour rechercher leur relation éventuelle avec le résultat de la FIV [I I] : il n'y a pas d'influence du nombre total de follicules préovulatoires développés sur les ovaires, ni du rang hiérarchique de ces follicules; une plus grande taille des follicules paraît favorable mais vraie avec le traitement exogène de stimulation $(3 \mathrm{ml}$ avec clomiphène et $I, 5 \mathrm{ml}$ avec clomiphène + HMG); à l'exception de l'œstradiol, des concentrations hormonales élevées dans le follicule (progestines, androgènes, gonadotropines) sont liées à un taux de fécondation supérieur;

(c) il existe également des facteurs liés au cycle ovarien ou à la patiente : la maturation folliculaire trop lente ou insuffisamment soutenue comme on peut l'apprécier par les niveaux périphériques de l'œstradiol) ou le déclenchement (injection de hCG de l'ovulation à un moment défavorable, sont à l'origine de certains échecs de fécondation. D'autre part, des ovocytes atrétiques sont plus souvent recueillis chez des patientes âgées ou présentant de fortes adhérences autour des ovaires.

Le résultat de la FIV est lié aux caractéristiques du spermogramme (tableau II). Dans notre population de couples FIVETE (indication essentiellement tubaire), la proportion de partenaires masculins dont le sperme est déficient, selon les critères usuels, est de I sur 4 . Il semble que les facteurs les plus influents du spermocytogramme soient la morphologie et le type de mouvement des gamètes.

\section{Fécondance des spermatozoïdes}

Afin de prendre en compte les divers paramètres du sperme (concentration, morphologie, mobilité) nous avons défini, provisoirement, un seuil arbitraire d'acceptation en FIVETE : présence d'au moins 5 millions de gamètes normaux et mobiles dans tout l'éjaculat. Pourtant, des échecs répétitifs avec le sperme de certains hommes prouvent que ces critères sont encore très grossiers; il devient urgent de caractériser les anomalies spécifiques des gamètes masculins compatibles avec la FIV.

Quand la fécondation a lieu, on constate que les chances de grossesse ne sont pas influencées par la qualité du sperme, puisque le nombre de grossesses cliniques pour Ioo patientes recevant des embryons in utéro est constant (tableau III voir page suivante).

La mise en contact des gamètes à 


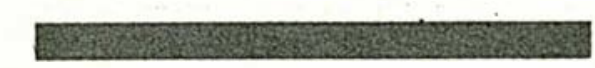

\begin{tabular}{|l}
\hline \multicolumn{4}{|c|}{ Tableau III } \\
INFLUENCE DE LA QUALITE DU SPERME SUR LES RESULTATS \\
DE LA FECONDATION ET DU TRANSFERT D'EMBRYONS
\end{tabular}

\section{REFERENCES}

14. Trouson A, Mohr L, Wood C, Leeton J. Effect of delayed insemination on in vitro fertilization, culture and transfer of human embryos. 7 Reprod Fert 1982; $64: 285-94$.

15. Testart J, Frydman R, de Mouzon J, Lassalle $\mathrm{R}$, Belaisch-Allart J. A study of factors affecting the success of human fertilization in vitro. Biol Reprod 1983; $28: 415-24$ et 425-31.

16. Nayudu P, Lopata A, Leung P, Johnston W. Current problems in human in vitro fertilization and embryo implantation. 7 Exp Zool 1983; 228 : 203-13.

17. Jones $H$, Acosta A, Andrews $M$ et al. There years of in vitro fertilization at Nolfolk. Fertil Steril $1984 ; 42: 826-34$.

18. Edwards R, Fishel S, Cohen J, et al. Factors influencing the success of in vitro fertilization for alleviating human infertility. I In Vitro Fertil Embryo Transfer 1984; $1: 323$.

19. Lopata A. Concepts in human in vitro fertilization and embryo transfer. Fertil Steril 1983; $40: 289-301$.

20. Feichtinger W, Kemeter P. Organization and computerized analysis of in vitro fertilization and embryo transfer programs. I In Vitro Fertil Embryo Transfer 1984, $1: 34-41$.

21. Trounson A. In vitro fertilization current topics. Exp Endocr 1984; $5: 43-73$.

22. Yovitch J, Stanger J, Yovitch J, Tuvik A. Quality of embryos from in vitro fertilization. Lancet 1984; $1: 457$.

23. Trounson $A$, Wood $C$. In vitro fertilization results, 1979-1982, at Monash University. I In Vitro Fertil Embryo Transf 1984; $1: 4247$.

24. Belaisch-Allart J, Frydman R, Testart J, et al. In vitro fertilization and embryo transfer program in Clamart, France. 7 In Vitro Fertil Embryo Transfer 1984; 1 : 5155 .

25. De Cherney A, Laufer $N$, Haseltine $F$, et al. Results of in vitro fertilization employind individualized ovulation induction by human menopausal gonadotropins. Fertil Steril 1984; 41 : (abstr) 96.

26. Frydman R, Eibschitz I, Papiernik E. Les indications médicales de la fecondation in vitro. médecine/sciences $1985: 1: 129-33$. des moments différents après leur recueil n'influence pas le résultat de la FIV : quand l'insémination in vitro a lieu avec un délai de 1,5 à 8 heures après le recueil de l'ovocyte ou de 0,5 à 6 heures après le recueil du sperme, on obtient la même proportion d'embryons par ovocyte inséminé [13].

\section{Facteurs méthodologiques}

Il est vraisemblable que l'incubation in vitro (pendant quelques heures) préalablement à l'insémination, est favorable quand l'ovocyte est recueilli immature [14]; mais la même pratique peut conduire à un vieillissement si l'ovocyte était mûr dès son recueil. Nous avons quelques difficultés pour distinguer les ovocytes matures des immatures : la distinction effectuée indirectement par le degré de dissociation des cellules périovocytaires (cumulus oophorus) est efficace après traitement avec le clomiphène [ I5] mais ne l'est plus si l'environnement folliculaire a été enrichi en FSH comme avec les traitements associant clomiphène et HMG. D'autre critères indirects, prenant en compte les teneurs du liquide folliculaire en certaines protéines, ont été proposés [I6]. Finalement on espère pouvoir, sans léser les chances de fécondation, dénuder les ovocytes dès leur recueil à l'aide d'enzymes; on pourrait ainsi reconnaître le stade méiotique, car ce sont les cellules périovocytaires qui gênent l'observation microscopique de l'ovocyte.

Le nombre de spermatozoïdes uti- lisés pour inséminer chaque ovocyte a progressivement diminué. Actuellement, plutôt qu'une concentration de I million par millilitre de milieu, on utilise moins de 100000 spermatozoïdes mobiles/ml. Dans nos conditions (ovocyte dans $0,5 \mathrm{ml}$ ), l'insémination est effectuée avec seulement 20000 spermatozoïdes, soit environ to 000 fois moins que le contenu d'un éjaculat normal. Il est presque toujours possible d'isoler cet effectif de gamètes même quand le sperme est très pauvre, mais il est parfois difficile de concentrer ces spermatozoïdes dans un très petit volume, utilisé pour l'insémination. De plus, la méthode usuelle de centrifugation-migration améliore la proportion des gamètes mobiles dans la fraction traitée mais ne modifie pas la proportion des gamètes à morphologie normale ou ayant effectué leur maturation nucléaire. Dans certains cas de spermes de qualité déficiente (oligo-, asthéno-, tératospermies), d'autres méthodes de préparation (migration ascendante, colonne d'albumine) peuvent être bénéfiques. Il n'existe pas, à ce jour, de procédés permettant d'améliorer, par l'action in vitro de certaines drogues, la fécondation des spermatozoïdes.

\section{Établissement de la grossesse}

Il est bien établi que l'introduction simultanée de plusieurs embryons facilite la survenue d'une grossesse avec un pourcentage élevé de grossesses multiples (tableau IV). De nombreuses équipes ont limité à trois le nombre maximum d'em- 
bryons replacés en un seul temps afin déviter la survenue de grossesses multiples dont on connait les complications obstétricales. Le transfert se pratique par voie cervicale; cette voie a pour avantage sa facilité, mais pour inconvénient le risque d'introduction de matériel étranger (sang ou mucus) et le risque de stimuler l'activité utérine. Cette.intervention ne nécessite pas d'anesthésie générale, mais parfois une neuroleptanalgésie lorsque le col est particulièrement tonique et présente une difficulté de passage.

Différents modèles de cathéters ont été proposés selon leur longueur, la taille, la localisation de l'orifice; ils peuvent être utilisés seul ou avec l'aide d'un mandrin qui permet de passer l'orifice interne du col cervical. La patiente est habituellement en décubitus dorsal, encore que certaines équipes préférent la position genu pectorale en fonction de la position de l'utérus. La quantité de liquide qui véhicule le ou les embryons est minime (10 à 20 microlitres). La patiente reste au repos, en position déclive, pendant I/2 h, puis peut sortir de l'hôpital.

Diverses médications ont été administrées pour soutenir la phase lutéale ou favoriser l'implantation mais aucune n'a fait la preuve de son efficacité. Plus que tout autre facteur, le taux de succès du transfert semble dépendre de la qualité de l'embryon ou des embryons replacés.

Toutefois le devenir des grossesses débutantes ne paraît pas lié au mọde de stimulation des ovaires puisque le taux de grossesses évolutives est remarquablement le même (70 à $74 \%$ ) quel que soit le traitement de la patiente (tableau $V$ ).

Comme l'indiquent Frydman et coll. dans ce même numéro [26], un élargissement des indications de la FIVETE existe déjà (stérilités idiopathiques, anticorps antispermatozoïdes chez la femme) et pourrait aller en augmentant (hypofertilités masculines, auto-anticorps anti-spermatozoïdes chez l'homme). Pour chacun de ces problèmes de stérilité, l'efficacité de la FIVETE devra être comparée à cellẹ des autres méthodes disponibles. Or, l'efficacité de la FIVETE devrait croître à court terme, essentielle-

\begin{tabular}{|c|c|c|c|c|}
\hline \multicolumn{5}{|c|}{$\begin{array}{l}\text { Tableau IV } \\
\text { SSESSE PAR TRANSFERT } \\
\text { DU NOMBRE D'EMBRYONS } \\
\text { SFĖRÉS IN UTÉRO }\end{array}$} \\
\hline \multicolumn{4}{|c|}{ nombre d'embryons transférés } & \multirow{2}{*}{$\frac{\text { [réf.] }}{[17]}$} \\
\hline $\begin{array}{c}20 \\
(153)\end{array}$ & $\begin{array}{c}23 \\
(147)\end{array}$ & $\begin{array}{c}29 \\
(72)\end{array}$ & $\begin{array}{l}35 \\
(57)\end{array}$ & \\
\hline $\begin{array}{c}15,7 \\
(655)\end{array}$ & $\begin{array}{c}27,8 \\
(255)\end{array}$ & $\begin{array}{l}36,2 \\
(58)\end{array}$ & - & [18] \\
\hline $\begin{array}{l}11,2 \\
(89)\end{array}$ & $\begin{array}{l}18,6 \\
(70)\end{array}$ & $\begin{array}{l}22,6 \\
(62)\end{array}$ & $\begin{array}{l}35,3 \\
(51)\end{array}$ & [19] \\
\hline $\begin{array}{l}11.7 \\
(34)\end{array}$ & $\begin{array}{l}30,0 \\
(30)\end{array}$ & $\begin{array}{l}36,4 \\
(11)\end{array}$ & $\begin{array}{l}- \\
-\end{array}$ & [20] \\
\hline $\begin{array}{l}18,3 \\
(115)\end{array}$ & $\begin{array}{l}26,0 \\
(100)\end{array}$ & $\begin{array}{l}30,0 \\
(60)\end{array}$ & - & [11] \\
\hline $\begin{array}{c}7 \\
(43)\end{array}$ & $\begin{array}{c}25 \\
(40)\end{array}$ & $\begin{array}{c}23 \\
(44)\end{array}$ & $\begin{array}{c}0 \\
(9)\end{array}$ & [21] \\
\hline $\begin{array}{c}15,7 \\
(1089)\end{array}$ & $\begin{array}{c}25,4 \\
(646)\end{array}$ & $\begin{array}{l}28,7 \\
(307)\end{array}$ & $\begin{array}{c}32,5 \\
(117)\end{array}$ & Total \\
\hline
\end{tabular}

Les chiffres entourés de parenthèses indiquent le nombre de patientes

\begin{tabular}{|c|c|c|c|c|}
\hline \multicolumn{5}{|c|}{$\begin{array}{c}\text { Tableau } V \\
\text { EVOLUTION DES GROSSESSES OBTENUES PAR FIVETE } \\
\text { SELON LE MODE D'INDUCTION DE L'OVULATION : } \\
\text { PROPORTION DE GROSSESSES EVOLUTIVES } \\
\text { PARMI LES GROSSESSES DEBBUTANTES }(a)\end{array}$} \\
\hline $\begin{array}{l}\text { aucun } \\
\text { traitement }\end{array}$ & CC & $\mathrm{CC}+\mathrm{HMG}$ & HMG & [réf.] \\
\hline $\begin{array}{c}38 / 52 \\
- \\
- \\
- \\
- \\
- \\
-\end{array}$ & $\begin{array}{c}127 / 166 \\
10 / 15 \\
20 / 33(b) \\
7 / 11 \\
- \\
- \\
-\end{array}$ & $\begin{array}{c}- \\
8 / 9 \\
35 / 46(b) \\
- \\
46 / 65 \\
- \\
-\end{array}$ & $\begin{array}{c}- \\
- \\
- \\
- \\
- \\
12 / 17 \\
70 / 105\end{array}$ & $\begin{array}{l}{[18]} \\
{[22]} \\
{[23]} \\
{[24]} \\
{[11]} \\
{[25]} \\
{[17]}\end{array}$ \\
\hline $\begin{array}{c}38 / 52 \\
(73,1 \%)\end{array}$ & $\begin{array}{l}164 / 225 \\
(72.9 \%)\end{array}$ & $\begin{array}{c}89 / 120 \\
(74.2 \%)\end{array}$ & $\begin{array}{l}82 / 123 \\
(66.7 \%)\end{array}$ & $\begin{array}{l}- \\
-\end{array}$ \\
\hline
\end{tabular}

(a) : on n'a pas tenu compte, dans la mesure du possible, des grossesses "biochimiques", limitées à une faible élévation du niveau de hCG plasmatique.

(b) : traitement le plus fréquent, mais non exclusif, pendant la prériode recensée. $C C$ : citrate de clomiphène.

$H M G$ : human menopausal gonadotropin 
Tableau VI

RO̊le DE LA RÉPONSE OVARIENNE DANS LE SUCCĖS DE LA FIVETE: RÉSULTATS THÉORIQUES A CHAQUE ÉTAPE,POUR 100 PATIENTES OPÉRÉES, EN FONCTION DU NOMBRE DE FOLLICULES PONCTIONNÉS.

\begin{tabular}{|c|c|c|c|c|c|}
\hline $\begin{array}{c}\text { nombre } \\
\text { de follicules } \\
\text { préovulatoires }\end{array}$ & $\begin{array}{c}\text { nombre } \\
\text { d'ovocytes } \\
\text { recueillis } \\
\text { ( } 70 \% \text { des } \\
\text { follicules) }\end{array}$ & $\begin{array}{c}\text { nombre } \\
\text { d'embryons } \\
\text { obtenus } \\
\text { ( } 70 \% \text { des } \\
\text { ovocytes) }\end{array}$ & $\begin{array}{c}\text { nombre } \\
\text { de transferts } \\
\text { d'embryon (s) }\end{array}$ & $\begin{array}{c}\text { nombre } \\
\text { de grossesses } \\
\text { (15 a } 30 \% \\
\text { des transferts } \\
\text { selon (le nombre } \\
\text { d'embryons) }\end{array}$ & $\begin{array}{c}\text { nombre } \\
\text { d'accouchements } \\
\text { ( } \sim 70 \% \text { des } \\
\text { grossesses) }\end{array}$ \\
\hline 1 & 70 & 50 & 50 & 8 & 6 \\
\hline 4 & 280 & 200 & $80(b)$ & 20 & 14 \\
\hline 8 & 560 & 400 & $90(c)$ & 25 & 18 \\
\hline $8(a)$ & 560 & 400 & $3 \times 90=270(d)$ & 40 & 28 \\
\hline
\end{tabular}

(a) avec cryopréservation des embryons.

(b) le plus souvent, transfert de deux embryons.

(c) le plus souvent, transfert de trois embryons.

(d) transfert successif des embryons un à un en admettant une survie après congélation de trois embryons sur quatre (75 \%).

ment grâce ̀̀ deux progrès techniques : l'obtention d'un plus grand nombre d'ovocytes fécondables par tentative et la possibilité de conserver par le froid les ovules ou surtout les embryons. La première amélioration devrait résulter de protocoles nouveaux de stimulation ovarienne, impliquant des hormones gonadotropes pures (FSH en particulier) et prenant date précocément (avant même les menstruations) en s'inspirant des événements naturels du cycle menstruel. $\mathrm{La}$ cryopréservation d'embryons humains a déjà permis quelques naissances après transfert, et des techniques efficaces et reproductibles seront bientôt connues. Ainsi deviendra t-il possible de procéder à plusieurs transferts d'embryons au cours de plusieurs cycles, chez la patiente ayant produit les ovocytes. Le tableau VI montre comment ces deux techniques influenceraient les résultats de la FIVETE : alors que 6 ou I4 \% des patientes opérées accouchent 9 mois plus tard, selon que leurs ovaires présentent un ou quatre follicules préovulatoires, cette proportion passe à $\mathrm{I} 8 \%$ pour huit follicules; surtout, en admettant que $75 \%$ des embryons survivent à la congélation, la même réponse ovarienne (huit follicules) couchements après cryopréservation et transfert un à un des embryons obtenus simultanément. Une autre technique permettrait d'augmenter le nombre d'embryons à transférer in utero pour un effectif folliculaire donné : il s'agit de la duplication provoquée en deux œufs vrais jumeaux de l'embryon obtenu en FIV. Cette technique n'a pas encore été utilisée, ses implications d'ordre éthique restant à approfondir.

Il est donc fortement probable que la FIVETE atteindra une efficacité comparable à celle de la fertilité naturelle dans les prochaines années (I 5 à $30 \%$ selon les auteurs). Dans - le même temps les techniques deviendront plus légères; déjà une proportion importante de patientes sont traitées en hôpital de jour et ne sont pas soumises à la coelioscopie ou l'anesthésie générale; avec notre protocole actuel de stimulation, trois patientes sur quatre sont soumises au recueil d'ovocytes le $\mathrm{I} 3^{\mathrm{e}}$ jour du cycle, entre 9 et $\mathrm{I} 2 \mathrm{~h}$; le contrôle hormonal du cycle précédant la FIVETE autoriserait la planification des interventions plusieurs mois à l'avance...

Il faut maintenant souhaiter que la FIVETE reste une méthode thérapeutique appliquée à des indications médicales précises et pratiquée dans des centres compétents

\section{Summary}

A few figures regarding in vitro fertilization and embryo transfer (IVFET): approximately 1500 children have been born throughout the world, and over I 00 in France. Current level of effectiveness: Io to I $5 \%$ live births following attempts to collect ova. Level of effectiveness predictable on a short-term basis: approaching or exceeding natural fecondity (15-30\%).

Factors related to improvement: obtaining a greater number of fertilizable ova and keeping fertilized ova at very cold temperatures. IVFET is now clearly one of the methods to be used against sterility.

\section{TIRES A PART}

J. Testart : Inserm U.187. Hôpital AntoineBéclère. 157 , rue de la porte-Trivaux. 92141 Clamart. 